\title{
L'AMAHUG dit non à la clause du besoin
}

Les membres de l'AMAHUG

En solidarité avec nos jeunes collègues, les membres de l'AMAHUG (Association des médecins-associés et médecins-adjoints des Hôpitaux Universitaires de Genève) protestent énergiquement contre la décision d'appliquer la clause du besoin qui limite l'ouverture de nouveaux cabinets pour les internes et chefs de clinique en fin de carrière hospitalière.

\section{Des besoins non précisés}

Quels sont les vrais besoins de la population en médecins? On ne dispose en Suisse d'aucun chiffres sérieux à ce sujet. La démographie médicale résiste notoirement aux projections qui sont au mieux imprécises et le plus souvent erronées. Comment prendre en compte la féminisation de la profession médicale et la diminution du temps de travail des médecins? A l'heure où les pays voisins qui ont connu un numerus clausus sévère il y a 15 ans manquent de médecins formés dans de nombreuses spécialités (anesthésie, obstétrique, notamment), cette mesure est-elle réellement judicieuse?

\section{Une mesure inefficace}

L'augmentation des coûts de la santé a potentiellement de nombreuses causes: l'augmentation des maladies chroniques, du nombre des personnes âgées, l'élargissement des prestations couvertes par la LAMal, les frais de gestion des caisses-maladie, l'augmentation du niveau de santé exigé par la population, le stress et ses conséquences dans une société en mutation, et le coût croissant des médicaments. Face à ces nombreux facteurs, quel est le poids de l'augmentation du nombre de médecins installés en pratique privée? Il n'existe à ce jour aucune étude sérieuse qui permette d'en quantifier l'impact réel sur les coûts de la santé.

Cette mesure serait applicable pendant trois ans seulement. Que se passera-t-il à l'issue de cette période? En quoi cette mesure temporaire empêchera-t-elle l'afflux de médecins de la Communauté européenne que nos politiques semblent redouter en raison de l'application des bilatérales?

\section{Une mesure injuste}

Cette mesure ne vise que nos jeunes collègues qui parviennent au terme d'une longue formation, durant en moyenne dix à quinze ans, et qui ne sont en rien responsables de la situation actuelle des coûts de la santé en Suisse. Il est dit que ce problème peut être réglé par l'augmentation du nombre de cadres dans les hôpitaux. Croit-on véritablement que l'on transforme du jour au lendemain un médecin qui désire ouvrir un cabinet en un médecin-cadre hospitalier?

\section{Une mesure au coût humain insupportable}

L'immense majorité des jeunes médecins a choisi ce métier pour être utile à leurs concitoyens dans l'épreuve de la maladie. Comment évaluer l'impact sur leur idéal et leur enthousiasme d'une mesure qui consiste tout simplement à leur dire: «Vous êtes inutiles!». Ce point nous tient particulièrement à cour en tant que formateurs au sein des Hôpitaux Universitaires de Genève, et nous craignons les effets d'une démotivation légitime de nos internes et chefs de clinique sur la qualité des soins dispensés dans notre institution.

\section{Ce n'est qu'un début ...}

On nous assure qu'il s'agit d'un moindre mal par rapport à une autre mesure dont on a beaucoup parlé, à savoir la suppression de l'obligation de contracter. Rappelons qu'il s'agit de la possibilité pour les caisses-maladie de choisir de ne rembourser que les médecins les «meilleurs marché», alors que rien ne garantit que ce soit les meilleurs médecins. Nous pensons au contraire que la clause du besoin n'est que la première d'une série de mesures destinée à mettre la profession médicale sous tutelle des assurances, sans que la population ait quoi que ce soit à dire dans ce débat.

Nous estimons que le problème des coûts de la santé en Suisse est une priorité politique absolue et sommes prêts à participer à une réflexion globale à ce sujet. En revanche, la clause du besoin est un expédient inefficace et insuffisant. En conséquence, nous nous associons à la manifestation organisée par nos jeunes collègues représentés pas l'AMIG (Association des Médecins des Institutions de Genève). 